\section{$\underset{\substack{\text { hommes } \\ \text { \& migrations }}}{ }$}

\section{Hommes \& migrations}

Revue française de référence sur les dynamiques

migratoires

$1284 \mid 2010$

Migrations et environnement

\title{
Rencontre avec Abed Azrié
}

\section{François Bensignor et Abed Azrié}

\section{CpenEdition \\ Journals}

\section{Édition électronique}

URL : http://journals.openedition.org/hommesmigrations/1271

DOI : 10.4000/hommesmigrations. 1271

ISSN : 2262-3353

\section{Éditeur}

Musée national de l'histoire de l'immigration

\section{Édition imprimée}

Date de publication : 1 mars 2010

Pagination : 190-195

ISSN : 1142-852X

Référence électronique

François Bensignor et Abed Azrié, «Rencontre avec Abed Azrié », Hommes \& migrations [En ligne], 1284 | 2010, mis en ligne le 29 mai 2013, consulté le 24 septembre 2020. URL : http:// journals.openedition.org/hommesmigrations/1271 ; DOI : https://doi.org/10.4000/ hommesmigrations.1271

Ce document a été généré automatiquement le 24 septembre 2020.

Tous droits réservés 


\title{
Rencontre avec Abed Azrié
}

\author{
François Bensignor et Abed Azrié
}

\section{NOTE DE L'ÉDITEUR}

Propos recueillis par François Bensignor

En janvier 2008, vous étiez invité à ouvrir les festivités de l'événement Damas capitale culturelle du monde arabe avec votre spectacle Suerte. Comment avez-vous perçu votre pays d'origine, vous qui vivez en France depuis plus de quarante ans?

La Syrie que j'ai retrouvée n'a rien à voir avec celle que j'avais quittée. J'ai découvert une presse libre, des radios et des télévisions privées, ce qui était impensable autrefois. Un système capitaliste - avec ses bons et ses mauvais côtés - s'est implanté en Syrie. Les musiciens français et espagnols qui m'accompagnaient m'ont affirmé qu'ils avaient rarement été aussi bien accueillis, d'abord sur le plan humain, et ont trouvé les conditions professionnelles et techniques excellentes.

En 2009, c'est à Damas qu'a été présentée la création de L'Évangile selon Jean, votre œuvre la plus ambitieuse, coproduite par le ministère syrien de la Culture et la Région Paca, par le biais de l'Orchestre des Jeunes de la Méditerranée. Une quarantaine de chanteurs et musiciens syriens et français étaient impliqués dans ce projet. Comment cette œuvre a-telle été reçue en Syrie?

En Syrie, ce sont les jeunes qui sont les plus sensibles à la musique que je propose. La génération des quadragénaires et surtout les quinquagénaires semblent être bloqués dans leurs goûts pour le tarab. Ils ont une idée préconçue de ce que doit être la musique. Dans les télévisions privées, dans les radios libres, j'ai rencontré des jeunes dans la vingtaine qui me disaient: "C'était un de nos rêves de vous voir. Nous écoutons votre musique ici.” Ça m'a fait grand plaisir. En 2001, nous avons enregistré les premières répétitions de L'Évangile avec l'Orchestre des Jeunes de la Méditerranée. Cet enregistrement a été piraté et un de ces jeunes gens m'a dit qu'il était allé spécialement en bus chercher cette pièce rare que détenait le disquaire d'un bourg de 1500 habitants près de Oms. Ces jeunes qui sont prêts à écouter toutes sortes de musiques ont également une sensibilité pour des formes nouvelles de musique 
orientale. Certains d'entre eux rejettent Oum Kalthoum, la grande référence musicale de leurs parents et de leurs grands-parents, parce qu'elle représente un système vétuste, une pensée conservatrice.

Lors de notre résidence d'une semaine à Damas pour préparer la première, beaucoup de jeunes voulaient assister à nos répétitions. Mais l'administration les repoussait, prétendant que c'était interdit. Nous répétions dans une salle du conservatoire, que nous devions traverser pour accéder à l'Opéra. Un jour, dans les couloirs, je croise une poignée de jeunes qui me disent qu'ils souhaitent assister à notre répétition, mais qu'on le leur interdit. Je les y ai invités en les priant seulement de nous prévenir, afin que l'on puisse les installer à un endroit où ils ne perturberaient pas la séance. Et chaque fois ils l'ont fait. Ils venaient à quatre ou cinq, assistaient à une heure de répétition et repartaient. C'était ma façon de travailler avec les musiciens qui les intéressait et, pour une fois, ils n'étaient pas confrontés à un mur de refus.

Comment ressentez-vous cette soudaine reconnaissance de votre musique dans votre pays d'origine?

Je ne suis pas quelqu'un qui a quitté son pays et souhaite y revenir. Mon pays n'est pas là-bas. Ce n'est pas la France non plus. Mon pays est là où nous sommes assis tous les deux dans cette pièce. Ce n'est pas très grand... Je n'ai aucune nostalgie de la Syrie. Je n'ai aucune envie d'être le fils prodigue qui revient chez lui. Rien ne me lie plus à ce pays. Mon lien avec la vie, avec un homme, une femme, un lieu, c'est la dynamique. Or je ne sens aucune dynamique avec la Syrie. Ma dynamique créative est européenne et essentiellement française. À une question d'un journaliste d'Al Jazira qui voulait savoir si je ne me sentais pas exilé en France, j'ai répondu qu'au bout de trois jours en Syrie, j'étais pris d'un profond ennui. Parce que nous ne partageons pas le même langage avec les gens, nous ne sommes pas sur la même longueur d'onde. Et finalement, après une quarantaine d'années, je découvre que le seul pays qui m'a donné et où l'on m'a aimé, c'est la France. C'est donc sans doute mon pays adoptif, mais c'est le meilleur parent que j'ai jamais eu.

Quand avez-vous commencé à envisager une adaptation musicale de L'Évangile selon Jean ?

Cette composition est liée à la mort de ma mère. J'ai commencé l'écriture de cette pièce, qui lui est dédiée, le lendemain de sa disparition, en 1982. Ma mère ne savait ni lire, ni écrire. Et elle s'émerveillait toujours de ma capacité de voyager grâce à mes connaissances. Elle m'a donné, en plus de l'amour d'une mère, cette exigence de toujours être autonome et responsable. En dehors de l'école, elle m'a obligé très jeune à aller travailler. Elle adorait le cinéma, allait au spectacle. Mais elle était aussi très croyante et l'exprimait par l'amour de l'autre. Mais à la fin de sa vie, elle n'allait plus à l'église. [...] Mon travail sur l'Évangile est en quelque sorte le réveil de l'amour que j'ai eu pour ma mère. Je l'avais un peu enfoui en venant à Paris et cette composition a ranimé la mémoire du lien que j'avais avec elle. J'ai passé des années à écrire le texte et, ce faisant, la musique se formait en moi. Ainsi sa mise en musique ne m'a demandé que 17 jours.

Comment avez-vous travaillé sur le texte?

J'ai suivi à la lettre l'écrivain qu'est Jean. Son texte original fait une centaine de pages. Je n'ai fait que découper, serrer, condenser les scènes qu'il a écrites, afin de mieux les mettre en musique. Je me suis ainsi trouvé devant 47 scènes. J'ai écrit 42 miniatures chantées et trois instrumentales. J'ai seulement ajouté trois préludes et 
une danse. Le premier prélude contient une dizaine de thèmes extraits de plusieurs scènes. J'ai inséré une "danse des tabernacles" à la fin de la première partie. Elle évoque cette fête juive commémorant le dressage des tentes dans le Sinaï, après la traversée de la mer Rouge. Cette partie musicale, dans laquelle l'orchestre occidental peut s'ébattre, allège un peu le chant. Le prélude à la deuxième partie prépare à la douleur, au procès, à la souffrance de la Passion. C'est une musique assez grave, jouée par l'ensemble oriental.

Le texte met en scène 35 personnages. Mais comme dans tous les livres sacrés, il n'y a pas de rôle féminin. Or, il m'était difficile d'écrire seulement pour des voix d'hommes. J'ai donc donné le rôle de l'Évangéliste à quatre femmes avec un homme. Parce que je voulais aussi ouvrir au registre des aigus afin de donner plus de clarté à la partie chantée. Chaque interprète chante toujours dans le même mode, si bien que lorsqu'une scène réunit quatre personnages, la partie chantée se déroule selon quatre modes différents. C'est un exercice difficile pour les chanteurs solistes, qui doivent aussi ornementer leur chant.

Comment vous confrontez-vous au caractère sacré des textes qui inspirent vos œuvres?

Pour moi, l'Évangile selon Jean comme l'Épopée de Gilgamesh sont de grands textes du patrimoine humain. Ces textes fondateurs n'ont rien à voir avec les "associations" qui les promeuvent aujourd'hui. Le Pape, chef de l'Église, et le Christ sont deux entités contraires. Paul a transformé le christianisme en une société de production. Il a établi un système politique avec ses lois. Or, le Christ, qui était un homme très moderne pour son temps, n'a jamais été un législateur, comme Moïse, Almorabi ou Mahomet. Aucun des quatre évangiles ne contient de législation. Le Christ était un homme plutôt métaphysique que physique et il n'a pas laissé de trace pour organiser une société. Sans Paul, il n'y aurait jamais eu ni Église, ni christianisme, lequel est aux antipodes du Christ.

Quand j'étais petit, j'ai été élevé par des prêtres syriaques, qui nous martelaient l'esprit avec l'Ancien et le Nouveau Testament: David et Goliath, l'Ecclésiaste, la Création... Je voyais dans mes rêves ce serpent qui venait visiter Ève. C'est terrible ! À 7 ou 8 ans, mes rêves venaient des images de l'iconographie religieuse. Mais à l'âge de 14 ans, j'ai eu un rejet global de tout cela. Je ne rejetais pas les textes, mais les sociétés qui les diffusaient. Et plus tard, lorsque j'en suis revenu aux véritables textes, j'ai constaté combien ils sont massacrés par ces gens-là.

J'ai compris que les textes originaux sont des piliers de l'histoire de l'humanité. Peu de gens connaissent le Livre des Morts des Égyptiens ou les écrits d'Akhenaton. Gilgamesh est intéressant, mais il existe des textes beaucoup plus graves et plus beaux, comme le poème babylonien de la création ou les cycles d'Ishtar, où l'on retrouve le Cantique des Cantiques. Il existe un patrimoine nourricier qui a évolué dans la région de la Mésopotamie, puis s'est étendu à la Méditerranée, jusqu'aux temps contemporains. Il est difficile de savoir aujourd'hui qui nous sommes sans passer par ces textes. Parce qu'ils sont à l'origine de nombreuses civilisations à l'Est comme à l'Ouest.

J'ai donc fait un retour vers ces textes de mon enfance. Et en les relisant, j'ai exercé un filtre afin de n'en garder que ce qu'ils ont de contemporain, éclairant la réalité des personnages qui nous sont proches. C'est leur côté charnel qui m'intéresse. Je veux les considérer comme des cousins, des connaissances. Et la musique que j'ai composée 
rejoint ce désir. En 2001, à la cathédrale de Gap, nous avons joué les premières pièces qui allaient constituer L'Évangile selon Jean. L'évêque de Digne est venu spécialement assister au concert. À peine avions-nous terminé qu'il est venu me dire : "Monsieur, je suis choqué par ce que je viens d'entendre! C'est une musique profane, elle est palpable, charnelle, alors qu'avec Bach ou Haendel nous avons l'habitude d'œuvres métaphysiques." Alors je l'ai remercié, parce que c'était exactement ce que j'avais besoin d'entendre. Je lui ai expliqué qu'en Orient, il n'existe pas de barrière entre musique sacrée et musique profane, que les paroles d'un hymne à la Vierge peuvent se transformer en une chanson d'amour entonnée sur la même mélodie au cabaret, devant un verre d'arak. Entre humain et divin, la frontière est extrêmement ténue. L'exemple des soufis, les mystiques musulmans, est là pour le démontrer. Les rois de Babylone, tout comme Gilgamesh, étaient aussi des prêtres, des médiateurs entre l'humain et le divin. On trouve cette phrase d'Ibn Arabî: “Dieu m'a demandé 'Qui es-tu?' Je lui ai répondu 'Je suis Toi !" Il n'y a pour moi rien de plus religieux comme de plus intime dans cette phrase. J'aimerais que les religions puissent revenir à ce genre de compagnonnage. C'est pourquoi j'ai voulu travailler l'Évangile à la manière d'un drame humain comme Ulysse ou une pièce de Shakespeare.

Pourquoi avoir voulu mettre en scène L'Épopée de Gilgamesh après L'Évangile selon Jean?

Depuis longtemps pour moi, Gilgamesh était un chantier ouvert. Je savais que c'était une œuvre sincère, qui méritait d'être plus aboutie. J'avais commencé ce travail en 1970. Pendant sept ans, je me suis plongé dans des recherches autour de cette grande épopée akkadienne, sur la mythologie mésopotamienne. J’ai lu pratiquement tous les textes religieux, métaphysiques, sociaux et politiques publiés en français et en arabe sur l'époque de Sumer et de Babylone. Mais ce temps "perdu" où l'on est entièrement engagé dans un travail de recherche ne l'est pas pour rien!

Avec le temps, cette Épopée de Gilgamesh apparaît comme l'œuvre fondatrice qui a déterminé la direction de votre travail pour les années à venir.

Ce travail a changé ma vie. J'ai compris qu'il existait autre chose avant les grandes religions monothéistes. Cela m'a permis de mieux appréhender la Bible, l'Ancien Testament, le Nouveau Testament et le Coran. Parce que j'ai trouvé des repères plus anciens. Humainement, spirituellement, métaphysiquement, philosophiquement, j'ai pu poser des questions beaucoup plus lointaines et construire une identité orientale, puis occidentale d'une autre façon.

Qu'est-ce qui a changé entre la version originale de 1977 et celle que vous avez recomposée pour la scène en 2009 ?

L'Épopée de Gilgamesh était ma première pièce de musique mélangeant l'inspiration orientale de mon enfance et la musique classique occidentale. Je l'avais conçue quasiment dans la fougue de l'adolescence, utilisant une profusion d'instruments. Le disque enregistré en deux jours en 1977 reste émouvant. Pour la version de scène, j'ai eu envie de réécrire les arrangements. Je n'y ai pas changé beaucoup de choses, excepté la partie des percussions. Dans sa version originale, L'Épopée de Gilgamesh se déroulait en trente-trois mouvements, dont seulement deux contenaient des percussions. Dans sa nouvelle version, c'est l'inverse : trente et un mouvements sont avec percussions et deux sans. J'ai transformé le quatuor de violoncelles, qui figurait dans la première version, en un trio à cordes (alto, violoncelle, contrebasse), qui offre un jeu de timbres plus intéressant. 
L'œuvre trouve ainsi une nouvelle respiration. Les arrangements sont plus fluides, l'orchestre mis en espace. Dans les parties chantées et parlées, la voix sonne différemment. La pièce prend le relief d'un charme nouveau, sa représentation s'accompagnant de projections de photos - sculptures ou dessins des personnages mythiques de l'épopée - sur lesquelles apparaissent les traductions du texte chanté. Une merveilleuse façon de marquer l'aboutissement de quatre décennies d'un travail raffiné, innovant et original.

\section{BIBLIOGRAPHIE}

\section{Discographie}

L'Évangile selon Jean (coffret 2 CD + DVD, Doumtak)

Mystic (Doumtak) + version CD + DVD

Suerte "Live in Berlin" (Doumtak)

Comptines pour enfants seulement (Doumtak)

Chants d'Amour et d'Ivresse (2 CD, Doumtak)

Venessia (Doumtak, $1^{\text {re }}$ édition 2000)

Omar Khayyam (Doumtak, $1^{\text {re }}$ édition 1999)

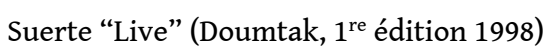

Lapis-Lazuli (Doumtak, $1^{\text {re }}$ édition 1996)

Aromates (Doumtak, $1^{\text {re }}$ édition 1990)

L'Épopée de Gilgamesh (Doumtak, $1^{\text {re }}$ édition 1977)

\section{RÉSUMÉS}

Compositeur d'œuvres profondes, inspirées de textes anciens essentiellement mystiques, Abed Azrié n'a renoué avec son pays natal, la Syrie, qu'en 2008, après plus de quarante années vécues en France. En 2009, sa création la plus ambitieuse, L'Évangile selon Jean, est jouée à l'Opéra de Damas, coproduite par le ministère syrien de la Culture. Le succès de cette pièce imposante pour voix solistes, chœurs et ensembles d'Orient et d'Occident, lui insuffle l'envie d'adapter pour la scène son chef-d'œuvre de jeunesse, L'Épopée de Gilgamesh. Fondatrice des bases d'une musique fusionnant savamment modes orientaux et harmonies occidentales, cette composition en 33 mouvements est accueillie avec enthousiasme trois soirs durant à l'auditorium de l'Institut du Monde Arabe à Paris en décembre 2009, puis en Allemagne, où elle est diffusée par la radio nationale. Abed Azrié évoque ce travail de quarante années, qui l'a construit comme musicien, 
compositeur et interprète à la voix de velours, mais également comme homme ouvert, sincère et intègre. 